\title{
ONE - IMAGE POZNAŃ ASTROLABE: PROJECT AND PROSPECTS
}

K. KURZYŃSKA, R. BARANOWSKI, P.A. DYBCZYŃSKI, A. GABRYSZEWSKA

Astronomical Observatory, A. Mickiewicz University, Poznań, Poland

AND

M. LEHMANN

Astrogeodynamical Observatory, Polish Academy of Sciences Kórnik, Poland

\begin{abstract}
A classical astrolabe has been modernised and reconstructed into a new fully automatic instrument equipped with a CCD camera and allowing observations at two different zenith distances: $45^{\circ}$ and $60^{\circ}$, respectively. The new instrument permits observations of objects fainter than those observable with the classical instrument. The work on the project is fairly advanced and regular observations are expected to begin in the nearest future. The paper reports results of the first observations and plans for the near future.
\end{abstract}

\section{Introduction}

The aim of this project is a continuation of astrometric observations in the "Post-Hipparcos Epoch". The stellar reference frame defined by Hipparcos will be very accurate (0.001 in positions, proper motions and paralaxes) but still will not be an inertial system. As this system rotates the determination precision of its rotation is not better than 0.00025 per year, so after several years its precision will be one order worse than the above mentioned. In this situation the astrometric observations from the Earth surface are still necessary provided the accuracy of instruments is improved. To continue the astrometric observations we have reconstructed a Danjon astrolabe (Danjon, 1960) which remains a very good astrometric instrument for the following reasons:

I. M. Wytrzyszczak, J. H. Lieske and R. A. Feldman (eds.),

Dynamics and Astrometry of Natural and Artificial Celestial Bodies, 557, 1997.

(C) 1997 Kluwer Academic Publishers. Printed in the Netherlands. 
- compact construction,

- movements around vertical axis only,

- lack of problems with deflection of the horizontal axis as in the case of the meridian circle,

- possibility of observation of various objects such as stars, radio-stars, planets, minor planets and even the Sun. (Chollet, 1991).

We have reconstructed the classical astrolabe from a two-images into a oneimage instrument applying a new optical solution and a CCD camera. It was the Astrolabe OPL No. 31, which had been in operation since 1982 to 1991 in Borowiec Observatory (Poland). During this period, primarily the Earth Rotation Parameters (ERP) were determined (Lehmann 1984, 1990, 1991). These observations were simultaneously used for catalogue works (Hefty and Lehmann, 1987; Lehmann, 1991). Our reconstructed astrolabe will not be used for the determination of the ERP but only for the determination of the coordinates of the stars and of Solar System objects (major and minor planets). At present, the astrolabe is working at the Poznan Astronomical Observatory but in the near future it will be moved back to the Borowiec Observatory, which is continuously controlled by the GPS and Satellite Laser Ranging System. This is just why we have decided to adapt it for astrometric observations in the forthcoming decade.

\section{Reconstruction of the Instrument}

The reconstruction of the astrolabe was performed aiming at two specific goals:

- a possibility of observations of objects fainter than those observable with the classical astrolabe,

- full automatization of the instrument and better accuracy of final results.

To increase the limit of the observable stellar magnitude, we have removed three optical elements from the classical instrument:

- the main prism,

- a polarising Wollaston prism which cuts about $40 \%$ of light,

- a small prism directing light to the ocular.

To improve the limit of stellar magnitude and the accuracy of observations we have applied the CCD camera as a detector. The removed optical elements were replaced with a new solution shown in Figure $1 \mathrm{~A}$.

The optical design of our new solution is based on the system of two reflecting prisms. The first prism defines (by the angle $\beta$ ) the working almucantar and the light from the celestial object going through the objective to the CCD camera is reflected from this prism. Its position is absolutely controlled by the second prism due to autocollimation through the reflec- 


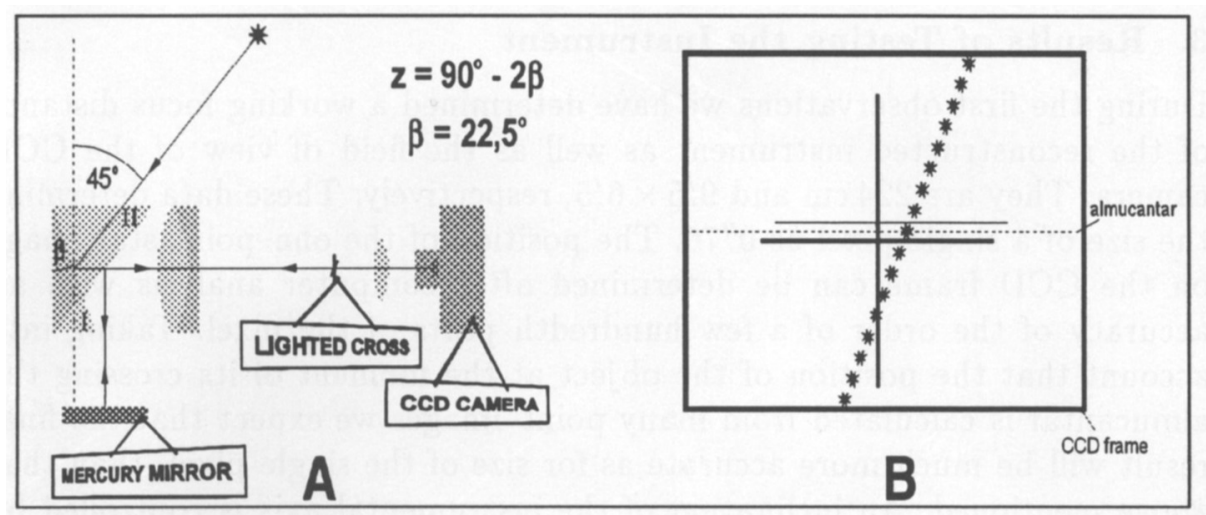

Figure 1. A: Optical solution of the astrolabe, B: Scheme of the autocolimation.

tion from the mercury mirror. A very important requirement is that these two prisms have to be rigidly connected. They are placed on a single plate which additionally hosts two small autocollimation mirrors, controlling the position of the whole two-prism system. To enable the autocollimation a crosshair is placed at the primary focus (see Figure 1A). This cross is enlightened by a set of laser diodes. In the CCD frame two images of the cross are seen: the direct and the reflected from the mercury mirror. The image of the zenith is exactly between the centers of these two crosses. The instrumental almucantar can be determined on the basis of the positions of the two horizontal lines (primary and reflected) of the cross, which is shown in Figure 1B. The same figure includes also the image of the observed star.

Independently, during the actual observation we record a CCD frame with the two crosses and another one with the trace of the star. In the process of reduction these two frames are superimposed for the determination of the star position in relation to the instrumental almucantar. In Figure $1 \mathrm{~B}$ the trace of a star is shown as a many-point "line", a consequence of the use of a shutter allowing to relate each position of the star to a specific time moment. Each moment of opening and closing the shutter is registered. The clock built in the computer is controlled by the GPS and in the future it will be controlled by the atomic clock at the Astrogeodynamical Observatory in Borowiec. Actually, the accuracy of time registration is better than $1 \mu \mathrm{sec}$. After this reconstruction, our instrument is now fully automatic and computer controlled. Both, the azimuth movement and reading are automatically performed according to the observational ephemeris. Before observation we have to input the ephemeris of the objects observed for a given night only. The azimuth, the time interval when the object is observed in the field of view, and the frequency of opening and closing the shutter are calculated automatically by the computer. 


\section{Results of Testing the Instrument}

During the first observations we have determined a working focus distance of the reconstructed instrument as well as the field of view of the CCD camera. They are $224 \mathrm{~cm}$ and $9.5 \times 6 ! 5$, respectively. These data determine the size of a single pixel as 0.76 . The position of the one-point star image on the CCD frame can be determined after computer analysis with an accuracy of the order of a few hundredth parts of the pixel. Taking into account that the position of the object at the moment of its crossing the almucantar is calculated from many point-images we expect that the final result will be much more accurate as for size of the single pixel, than that above mentioned. An inclination of the instrumental axis is controlled by the position of the primary and reflected crosses on the CCD frame. We have examined the variation of this axis in the $360^{\circ}$ range of the azimuth. We found a good stability of this axis with an amplitude of inclination of no more than \pm 10 "' 0 .

The second aim of the project was the extension of the observation to fainter objects than it had been possible with the classical astrolabe. Up to now we have observed stars of 8.9 magnitude. The quality of the image on the CCD frame allows us to expect a possibility of observing even fainter objects. We have at our disposal two sets of prisms for the observations at $45^{\circ}$ and $60^{\circ}$ of the zenith distance, respectively. The latter set will be used for observations of the Solar System objects, possible at small altitudes in our geographical location. With the two sets of prisms we will be able to determine for two different zenith distances the absolute declinations of the observed objects (Krejnin, 1968).

Acknowledgement. The authors wish to thank Dr. Fernand Chollet for cooperation in the design of the instrument optics. This research was supported by the KBN grant No. 2 Z600 00106.

\section{References}

Chollet, F.: 1991, "Report of Commission 8: Positional Astrometry", Trans. IA U, XXIB, preprint.

Danjon, A.: 1960, "The impersonal astrolabe", in: Telescopes (G.P. Kuiper, B.M. Middlehurst, eds), The University of Chicago Press, p.115.

Hefty, J. and Lehmann, M.: 1987, "Two catalogues of FK4 Right-ascension connections and their application", Bull. Astron. Inst. Czech. 38, 24.

Krejnin, E.I.: 1968, 'The absolute determination of star declinations with instruments without precise graduated circles', Astron. Zh. 45, 447 (in Russian).

Lehmann, M.: 1984, "The programme of observations with Danjon Astrolabe at Astronomical Latitude Observatory in Borowiec", Wiss. Z. Techn. Univ. Dresden, 33, 117.

Lehmann, M.: 1990, "Differences in changes of Borowiec geographical coordinates caused by the replacement of FK4 Catalogue by FK5", in: Inertial Coordinate System on the Sky, IAU Symposium 141 (J.H. Lieske, V.K. Abalakin, eds), Kluwer, Dordrecht.

Lehmann, M.: 1991, "Astronomical observations of variations of Borowiec geographical coordinates in the years 1982-1990", Artif. Satell. 26, 277. 